\title{
T80Cam: The wide field camera for the OAJ 83-cm telescope
}

\author{
A. Marín-Franch*a ${ }^{*}$ K. Taylor ${ }^{b}$, J. Cepa $^{\mathrm{c}}$, R. Laporte ${ }^{\mathrm{d}}$, A. J. Cenarro ${ }^{\mathrm{a}}$, S. Chueca ${ }^{\mathrm{a}}$, D. Cristobal- \\ Hornillos $^{\mathrm{a}}$, A. Ederoclite ${ }^{\mathrm{a}}$, N. Gruel ${ }^{\mathrm{a}}$, J. Hernández-Fuertes ${ }^{\mathrm{a}}$, A. López-Sainz ${ }^{\mathrm{a}}$, R. Luis-Simoes ${ }^{\mathrm{a}}, \mathrm{M}$.

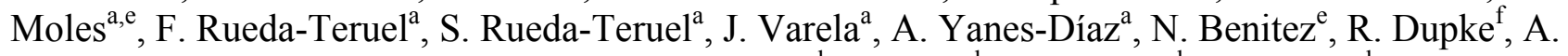 \\ Fernández-Soto $^{\mathrm{g}}$, C. Mendes de Oliveira ${ }^{\mathrm{b}}, \mathrm{G}$. Sims ${ }^{\mathrm{h}}$, L. Sodré Jr ${ }^{\mathrm{b}}$, K. Toerne ${ }^{\mathrm{h}}$ \\ ${ }^{a}$ Centro de Estudios de Física del Cosmos de Aragón (CEFCA), Plaza San Juan 1, Planta 2, E-44001 \\ Teruel, Spain; ${ }^{b}$ Universidade de Sao Paulo, IAG, Rua do Matao, 1226, Sao Paulo, 05508-900, \\ Brasil; ' Instituto de Astrofísica de Canarias, 38200 La Laguna, Tenerife, Spain; 'Divisao de \\ Astrofísica, Instituto Nacional de Pesquisas Espaciais, Brazil; ${ }^{\mathrm{e} I n s t i t u t o ~ d e ~ A s t r o f i ́ s i c a ~ d e ~ A n d a l u c i ́ a, ~}$ \\ Consejo Superios de Investigaciones Científicas (CSIC), C/ Camino Bajo de Huétor 50, E-18008 \\ Granada, Spain; fObservatorio Nacional, Rua Gal. Jose Cristino, 20921-400, Rio de Janeiro, Brazil; \\ ${ }^{\mathrm{g}}$ Instituto de Física de Cantabria (CSIC-UC), E-39005, Santander, Spain; ${ }^{\mathrm{h}}$ Spectral Instruments, Inc., \\ 420 N. Bonita Ave., Tucson, AZ 85745, USA
}

\begin{abstract}
The Observatorio Astrofísico de Javalambre (OAJ) is a new astronomical facility located at the Sierra de Javalambre (Teruel, Spain) whose primary role will be to conduct all-sky astronomical surveys. The OAJ facility will have two wide-field telescopes: the JST/T250; a $2.55-\mathrm{m}$ telescope with a $3^{\circ}$ diameter field of view (FoV), and the JAST/T80; an $0.83-\mathrm{m}$ telescope with a $2^{\circ}$ diameter FoV. First light instrumentation is being designed to exploit the survey capabilities of the OAJ telescopes. This paper describes the T80Cam, a wide-field camera that will be installed at the Cassegrain focus of the JAST/T80. It is equipped with an STA 1600 backside illuminated detector. This is a $10.5 \mathrm{k}-$ by- $10.5 \mathrm{k}, 9 \mu \mathrm{m}$ pixel, high efficiency CCD that is read from 16 ports simultaneously, allowing read times of 20 s with a typical read noise of 6 electrons (rms). This full wafer CCD covers a large fraction of the JAST/T80's FoV with a pixel scale of $\sim 0.50 "$ /pixel. T80Cam will observe in the wavelength range 330-1000nm through a set of 12 carefully optimized broad-, intermediate- and narrow-band filters. The camera is intended for surveys with the JAST/T80 telescope, starting with the planned J-PLUS (Javalambre Photometric Local Universe Survey), a multi-band photometric all-sky survey that will be completed in about 2 years and will reach $\mathrm{AB} \sim 23 \mathrm{mag}(5 \sigma$ level) with the SDSS filters.
\end{abstract}

Keywords: Instrumentation, CCD Camera, wide field, Observatorio Astrofísico de Javalambre.

\section{INTRODUCTION}

The Centro de Estudios de Física del Cosmos de Aragón(CEFCA) is a new research institution located at Teruel (Spain). CEFCA is responsible for the definition, design, construction and operation of the Observatorio Astrofísico de Javalambre $^{1,2}(\mathrm{OAJ})$, a new robotic observatory located at the Sierra de Javalambre (Teruel, Spain) whose primary role will be to conduct all-sky astronomical surveys. The OAJ facility will have two wide-field telescopes: the JST/T250; a 2.55-m telescope with a $3^{\circ}$ diameter FoV, and the JAST/T80; an 0.83-m telescope with a $2^{\circ}$ diameter FoV. First light instrumentation is being designed to exploit the survey capabilities of the OAJ telescopes, starting with the planed J-PAS and J-PLUS surveys.

J-PAS (Javalambre-PAU Astrophysical Survey) will be carried out with the JST/T250 telescope and JPCam ${ }^{3}$. J-PAS is an innovative photometric survey of the northern sky using an unprecedented system of 56 filters ${ }^{4}, 54$ narrow-band (FWHM $\sim 13.8 \mathrm{~nm}$ ), plus 2 broad-band filters, covering the 330-1000nm wavelength range, over more than 8000 square degrees. The J-PAS survey will reach $\mathrm{AB}=22-23$ within 3 arcsec aperture at $5 \sigma$ level, depending on the wavelength. Apart from these 56 filters, an additional broad-band filter will be used for deep imaging.

*amarin@cefca.es; phone+34 978221266 - Ext 1125; www.cefca.es

Ground-based and Airborne Instrumentation for Astronomy IV, edited by lan S. McLean, Suzanne K. Ramsay, Hideki Takami, Proc. of SPIE Vol. 8446, 84466H · ( ) 2012 SPIE · CCC code: 0277-786X/12/\$18 · doi: 10.1117/12.925422 
Together with the main J-PAS survey, the collaboration is carrying out the J-PLUS (Javalambre Photometric Local Universe Survey). This survey will observe the same area as the J-PAS through a set of 12 carefully optimized broad-


SDSS filters. This survey will be used to do the calibration tasks for the main J-PAS survey. In order to do that J-PLUS will create a set of thousands of stars which will be used as secondary photometric standard stars to calibrate J-PAS ${ }^{5}$.

J-PLUS will be carried out using JAST/T80 telescope and its first light instrument, T80Cam, a wide field camera to be installed at the JAST/T80 Cassegrain focus. T80Cam is intended for surveys with the JAST/T80. Table 1 summarizes nominal performances of T80Cam. The instrument consists of two main subsystems (Figure 1): the filter and shutter unit (FSU) and the camera subsystem. The FSU holds two removable filter wheels and the shutter. The camera subsystem, below, comprises the cryostat, the cooling and vacuum systems, the CCD, an optically powered entrance window and the detector electronics. These two subsystems are described in the following.

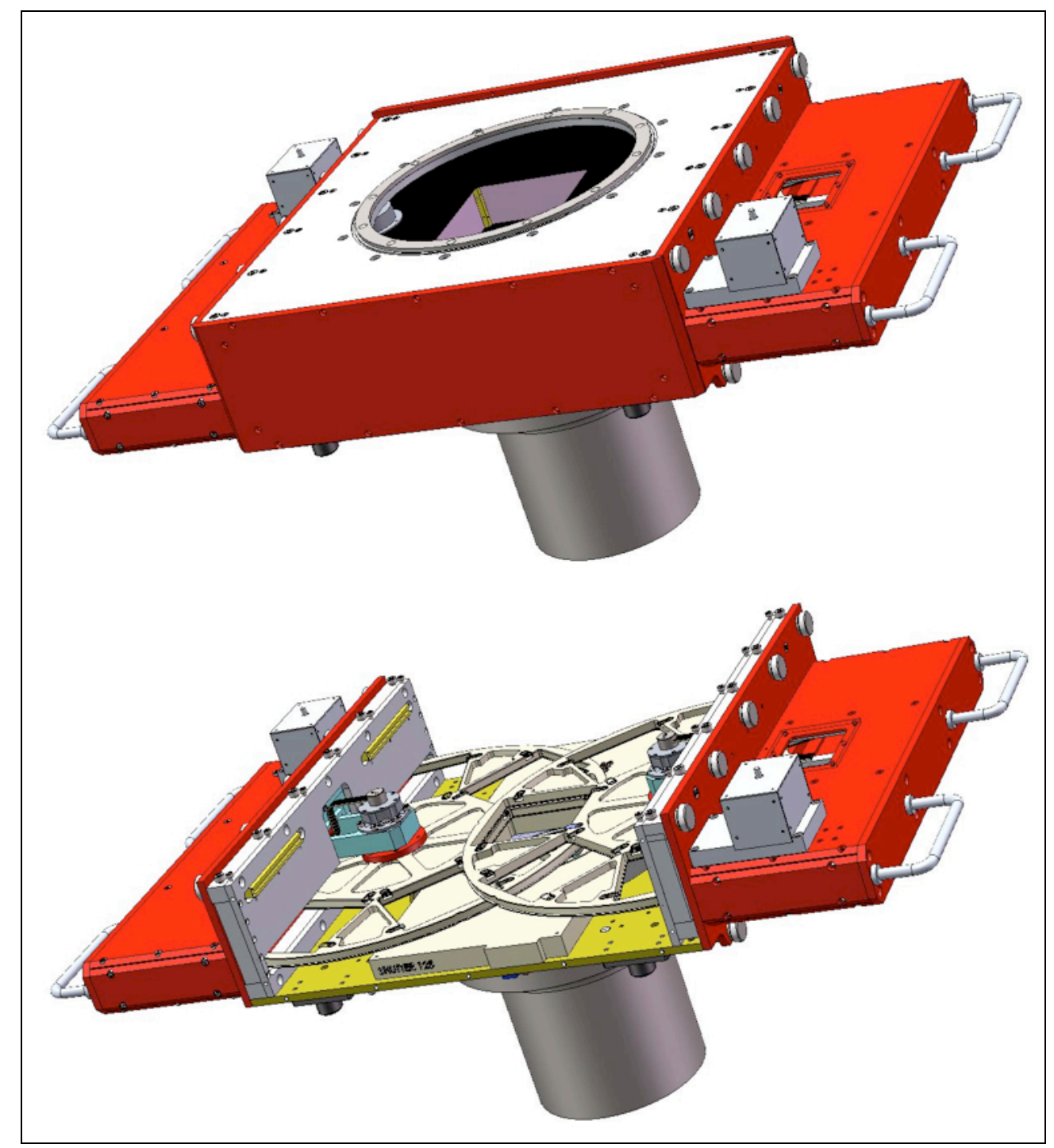

Figure 1. T80Cam preliminary design. Upper panel shows the complete assembled instrument. The two main subsystems can be clearly identified. The top part of the instruments represents the FSU containing the shutter and the two filter wheels. The cylindrical-shaped object underneath the FSU represents the camera subsystem. Lower panel shows the same view of T80Cam after cover removal to show the two filter wheels and the shutter. 
Table 1. T80Cam nominal performance.

\begin{tabular}{|l|l|}
\hline FoV & $\begin{array}{l}\varnothing=1.7^{\circ} \text { (full performance) } \\
\varnothing=2.0^{\circ} \text { (reduced performance) }\end{array}$ \\
\hline EE50 & $\emptyset=6 \mu \mathrm{m}$ \\
EE80 & $\varnothing=13 \mu \mathrm{m}$ \\
\hline CCD format & $10,580 \times 10,580 \mathrm{pix}$ \\
& $9 \mu \mathrm{m} / \mathrm{pix}$ \\
\hline Pixel scale & $0.5 \% / \mathrm{pix}$ \\
\hline FoV coverage & $2.1 \mathrm{square}$ degrees (fill factor $\sim 70 \%$ ) \\
\hline Read out time & $<20 \mathrm{~s}$ \\
\hline Read out noise & $<6 \mathrm{e}-/ \mathrm{pixel}$ \\
\hline Full well & $>70,000 \mathrm{e}^{-}$ \\
\hline CTE & $>0.99995$ \\
\hline Dark current & $>0.001 \mathrm{e}^{-} / \mathrm{pixel} / \mathrm{s}$ \\
\hline Number of filters & 12 \\
\hline
\end{tabular}

\section{CAMERA SYSTEM}

The camera system is an 1110 S camera manufactured by Spectral Instruments (Tucson, AZ, USA). It is equipped with a grade-1 STA 1600 backside illuminated CCD, a $10.5 \mathrm{k}-$ by-10.5k, $9 \mu \mathrm{m}$ pixel high efficiency CCD. As it can be seen in Figure 2, this full wafer CCD covers a large fraction of the JAST/T80's FoV with a pixel scale of $\sim 0.50 " /$ pixel.

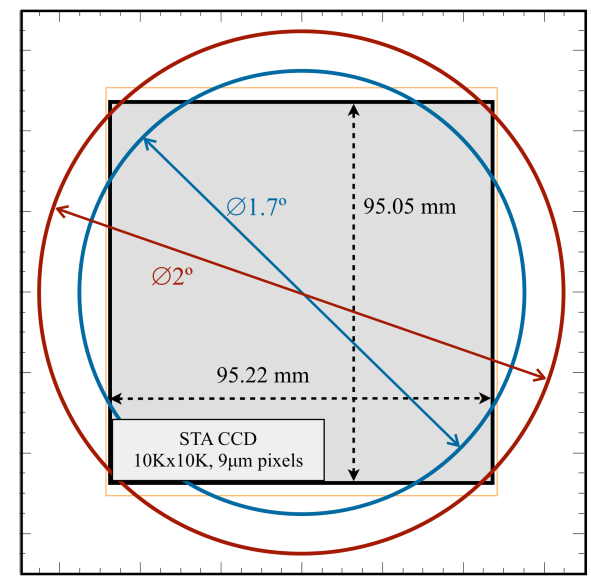

Figure 2. T80Cam's full (inner circle) and reduced (outer circle) performances optical FoV. The grey square represents the STA 1600 CCD covering a large fraction of the JAST/T80 telescope's FoV.

The control electronics allow for regions of interest (RoI) to be selected with consequent shortening in the read time. Image acquisition software provides FITS images with headers containing all the information relevant to the camera and telescope operation. Together with the CCD and its control electronics, the camera system is completed with a powered cryostat entrance window and cooling and vacuum systems.

\subsection{CCD}

The STA 1600 CCD has a format of 10580-by-10560, with $9 \mu \mathrm{m}$ pixels. The backside illuminated version has been selected for increased sensitivity and UV response. This large format CCD has an image area of $95.05 \mathrm{x} 95.22 \mathrm{~mm}$. It is read from 16 ports simultaneously. A readout speed of $400 \mathrm{kHz}$ with 16 ports allows read times of $<20$ s with a typical read noise of $<6$ electrons (rms). CCD performance is listed in Table 1. The CCD has a broadband AR coating for optimized performance from 380 to $850 \mathrm{~nm}$. 


\subsection{Cooling System}

The sensor is cryo-cooled to an operating temperature between $-100^{\circ} \mathrm{C}$ and $-110^{\circ} \mathrm{C}$ with a cryo-tiger refrigeration system, a closed-cycle Joule-Thomson effect cryogenic refrigerator system. The cryo-tiger cooling system has no moving parts in the cold end (nearly no vibrations), operates in any orientation and requires almost no maintenance.

\subsection{Vacuum System}

The chamber will be evacuated to a level of $10^{-4}$ Torr using a turbo dry vacuum pump. The pressure in the camera head is constantly monitored by the electronics hardware. Alarms are output if the camera head vacuum needs to be refreshed. This is expected to happen about once per 6-12 months.

\subsection{Entrance Window}

The camera entrance window forms the last element of the JAST/T80 field corrector, and together with the filters, it is part of the telescope optical design optimization. The window is a $10 \mathrm{~mm}$ thick, weakly powered field-flattener with an $8 \mathrm{~mm}$ distance between its inner surface and the focal plane. The entrance window will be made of quartz glass with a plano-concave shape.

\section{FILTER AND SHUTTER UNIT}

The FSU is being designed and manufactured by the Instituto Nacional de Pesquisas Espaciais (INPE) and CEFCA. It includes the filter unit, the shutter and the cryostat support flange. The later allows for fine alignment of the camera system with respect to the telescope optical axis. FSU main components are described next.

\subsection{Shutter}

The shutter has been acquired through the Argelander Institut fur Astronomie. It has a $125 \mathrm{~mm}$ clear aperture and is placed downstream between the filter wheels and the detector focal plane and presents no vignetting of the detector. This shutter offers an exposure homogeneity $<1 \mathrm{~ms}$ over the full field of view. It will be activated and synchronized with the operation of the detector read electronics and the filter wheel.

\subsection{Filter Unit}

The FSU holds two removable filter wheels, each one capable of holding 6 filters plus an empty position. Filters in both wheels present no vignetting of the detector. Filter wheels control is performed using stepper motors and absolute rotary encoders.

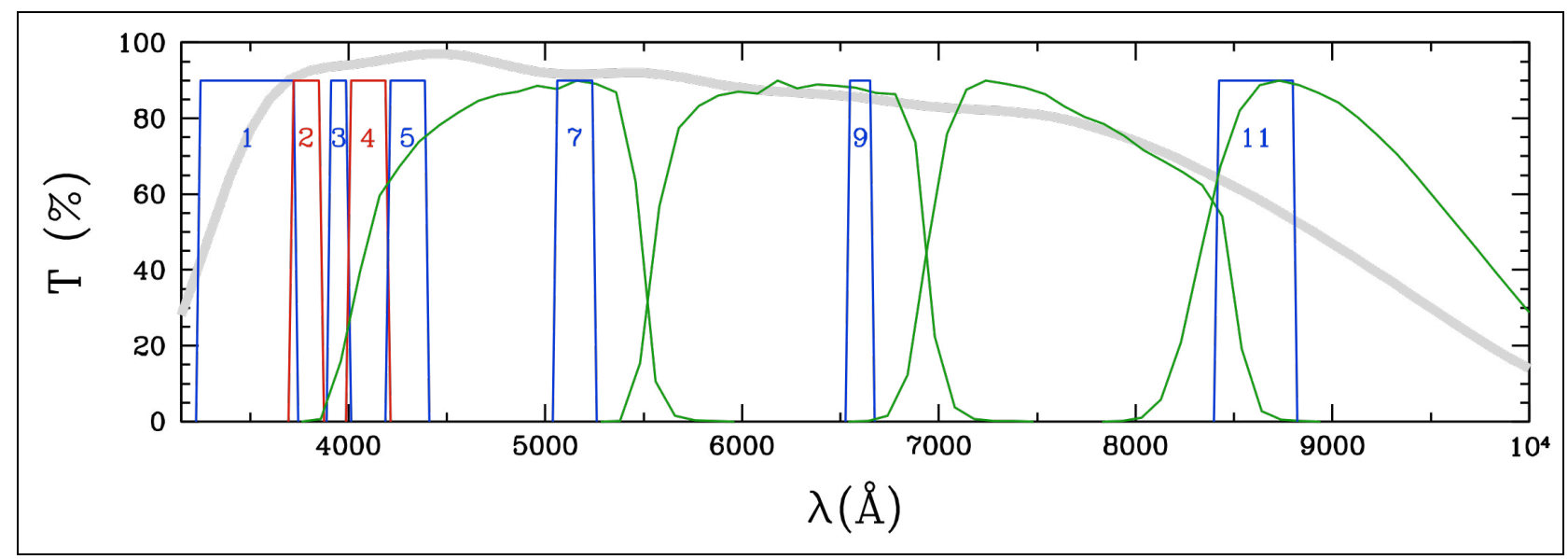

Figure 3. J-PLUS filter system. Broad-band filters are Sloan g', r', i' and z'. Narrow-band filters are custom designed JPLUS filters. Thick grey line represents the expected CCD quantum efficiency. 
The T80Cam is optimized to operate in the wavelength range 330-1000nm through a set of 12 carefully optimized broad, intermediate- and narrow-band filters ${ }^{4,5}$. Figure 3 shows the transmission curves of the 12 filters that T80Cam will mount to carry out the J-PLUS survey.

\subsection{Tip/tilt adjustment of the detector focal plane}

In order to align the instrument with the telescope's optical axis, a tip/tilt of the focal plane is necessitated to accommodate tolerances in both the telescope/FSU and the FSU/dewar interfaces. T80Cam incorporates 4 tip/tilt manual adjustments for what is anticipated to be a commissioning-only alignment. Figure 4 shows the preliminary design of the tip/tilt adjustment system.

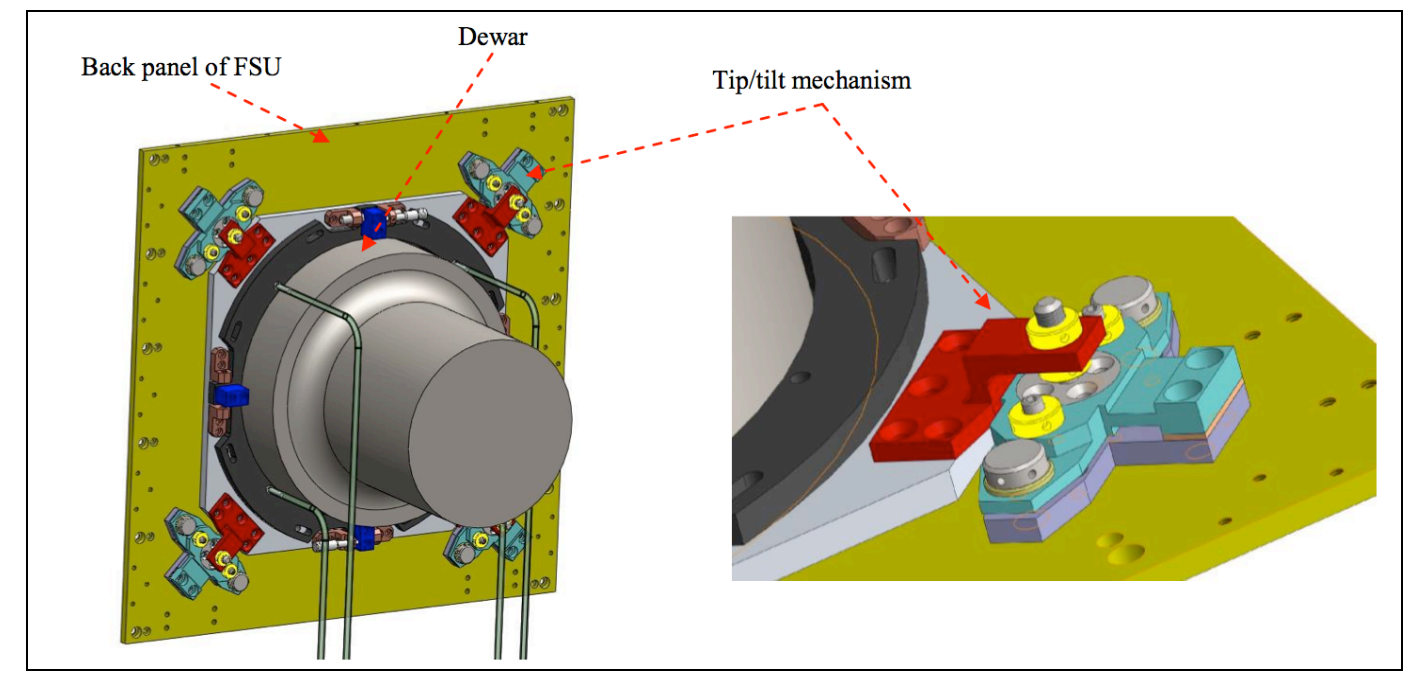

Figure 4. FSU/camera system interface plate showing the location of 4 tip/tilt adjusters. Right: Detailed view of tip/tilt adjuster.

\section{T80CAM OPTICAL PERFORMANCES}

The polychromatic encircled energy corresponding to the center $(0 \mathrm{deg})$, mid $(0.5 \mathrm{deg})$ and edge $(0.85 \mathrm{deg})$ of the full FoV is represented in Figure 5. The diffraction limited encircled energy (EE) formed by the same elements is shown for comparison. The calculation of the diffractive EE takes account of the obscuration by the telescope's M2 mirror and is an average over the full spectral range $(330-1000 \mathrm{~nm})$.

The spot diagrams of the JAST/T80 + T80Cam are represented in Figure 6 for field angles of $0^{\circ}, 0.25^{\circ}, 0.5^{\circ}$ and $0.85^{\circ}$.

\section{STATUS AND SCHEDULE}

The camera subsystem mechanical design is finished and mechanical parts are about to be fabricated. The STA 1600 CCD is already manufactured and is now in the thinning facility. All the electronics boards for the camera subsystem have been assembled and control software is under development. The FSU passed its PDR in March 2012, and is close to final design. J-PLUS filters are being designed now and manufacture will start within the next few weeks. According to our current schedule, T80Cam will be fully integrated and ready for scientific operation in December 2012. 


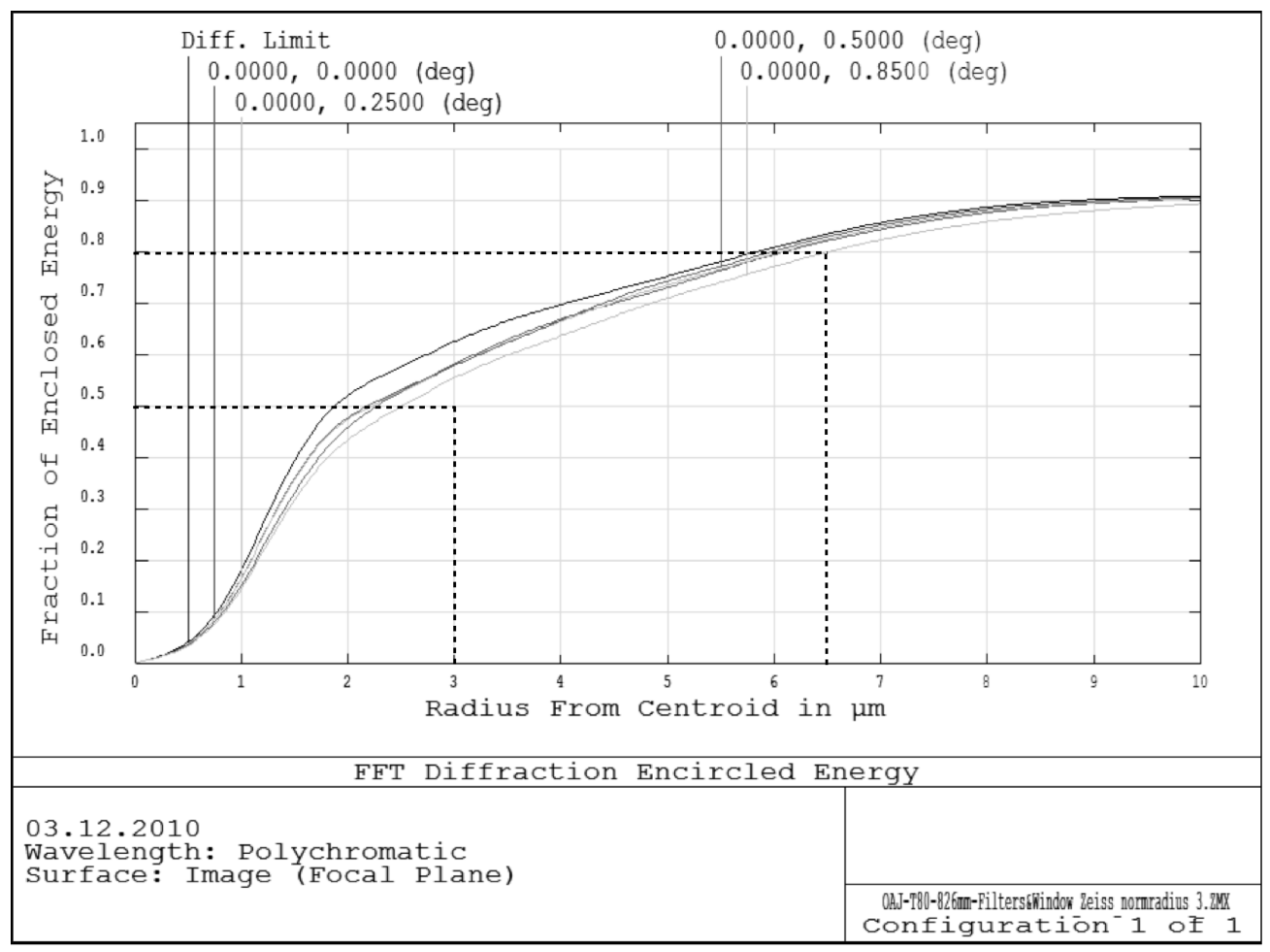

Figure 5. Diffractive Encircled Energy. EE50 and EE80 performances of the JAST/T80 + T80Cam design are marked.

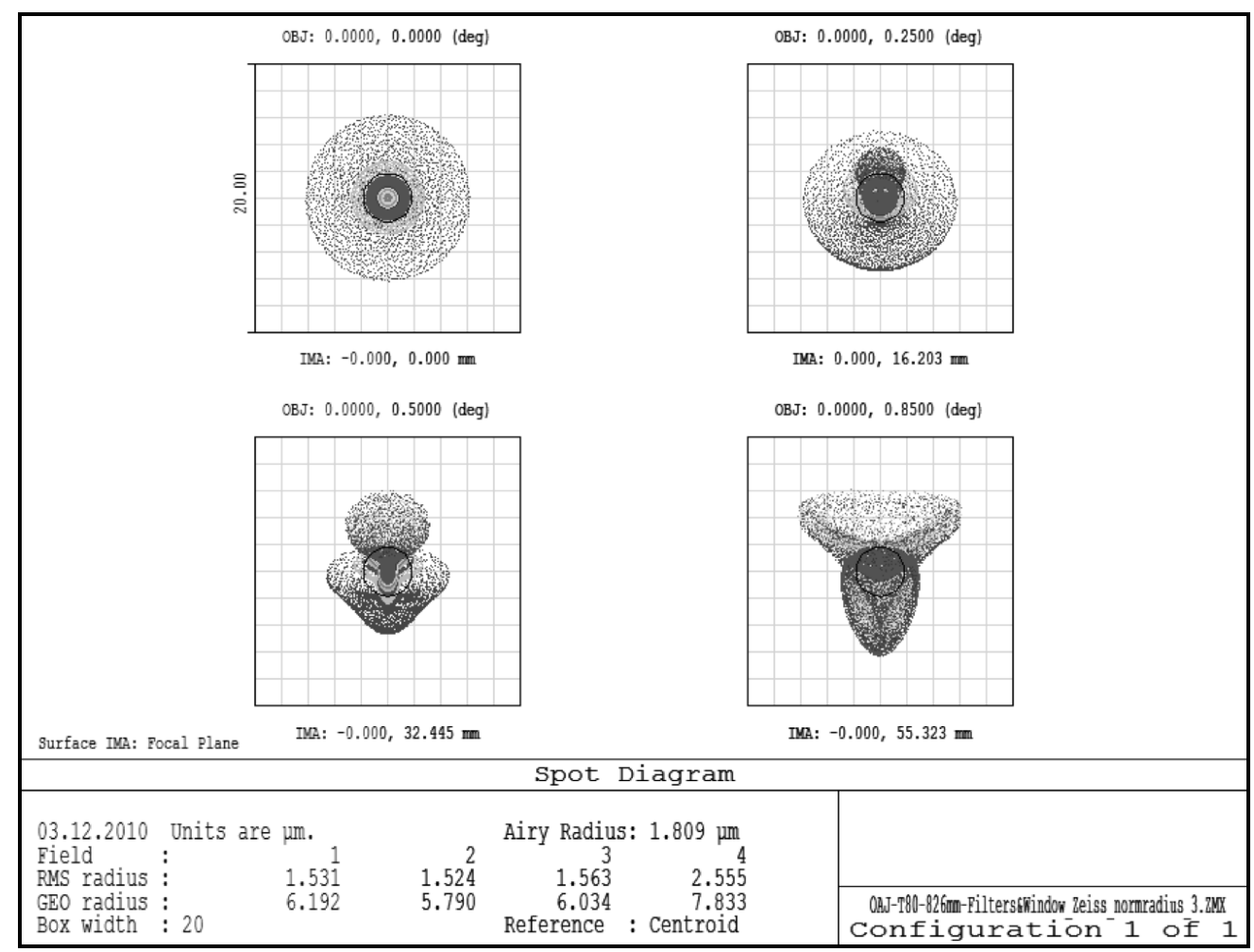

Figure 6. Spot Diagram for field angles of $0^{\circ}, 0.25^{\circ}, 0.5^{\circ}$ and $0.85^{\circ}$. 


\section{ACKNOWLEDGMENTS}

The OAJ is funded by the Fondo de Inversiones de Teruel, supported by both the Government of Spain (50\%) and the regional Government of Aragón (50\%). This work has been partially funded by the spanish Ministerio de Ciencia e Innovación through the PNAYA (under grants AYA2006-14056 and AYA2010-22111-C03-02) and through the ICTS 2009-14, and the Fundación Agencia Aragonesa para la Investigación y Desarrollo (ARAID). We would like to thank Vicente Sánchez and Jorge Sánchez-Capuchino for their significant contribution to the camera preliminary design review.

\section{REFERENCES}

[1] A. J. Cenarro et al., "The Javalambre Astrophysical Observatory project", Proc. SPIE 7738 (2010).

[2] A. J. Cenarro et al., "The Observatorio Astrofisico de Javalambre: goals and current status", Proc. SPIE 8448 (2012).

[3] K. Taylor et al., "JPCam: A 1.2Gpixel camera for the J-PAS survey", Proc. SPIE 8446 (2012).

[4] A. Marin-Franch et al., "Design of the J-PLUS and J-PAS filter Systems", Proc. SPIE 8450 (2012).

[5] N. Gruel et al., "Calibration plan for J-PAS and J-PLUS surveys", Proc. SPIE 8448 (2012). 